\title{
Genetic diversity of Escherichia coli in gut microbiota of patients with Crohn's disease discovered using metagenomic and genomic analyses
}

Alexander V. Tyakht ${ }^{1,2,3^{*}}$ (D), Alexander I. Manolov', Alexandra V. Kanygina², Dmitry S. Ischenko ${ }^{1,2}$, Boris A. Kovarsky', Anna S. Popenko', Alexander V. Pavlenko', Anna V. Elizarova², Daria V. Rakitina', Julia P. Baikova', Valentina G. Ladygina', Elena S. Kostryukova ${ }^{1,2}$, Irina Y. Karpova' ${ }^{1}$, Tatyana A. Semashko ${ }^{1,2}$, Andrei K. Larin ${ }^{1}$, Tatyana V. Grigoryeva ${ }^{4}$, Mariya N. Sinyagina ${ }^{4}$, Sergei Y. Malanin ${ }^{4}$, Petr L. Shcherbakov ${ }^{5}$, Anastasiya Y. Kharitonova ${ }^{6}$,

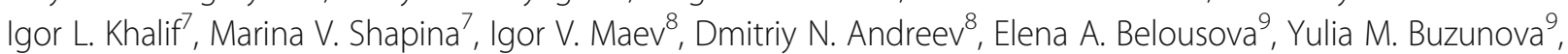
Dmitry G. Alexeev ${ }^{1,2}$ and Vadim M. Govorun ${ }^{1,2,10}$

\section{Abstract}

Background: Crohn's disease is associated with gut dysbiosis. Independent studies have shown an increase in the abundance of certain bacterial species, particularly Escherichia coli with the adherent-invasive pathotype, in the gut. The role of these species in this disease needs to be elucidated.

Methods: We performed a metagenomic study investigating the gut microbiota of patients with Crohn's disease. A metagenomic reconstruction of the consensus genome content of the species was used to assess the genetic variability.

Results: The abnormal shifts in the microbial community structures in Crohn's disease were heterogeneous among the patients. The metagenomic data suggested the existence of multiple E. coli strains within individual patients. We discovered that the genetic diversity of the species was high and that only a few samples manifested similarity to the adherent-invasive varieties. The other species demonstrated genetic diversity comparable to that observed in the healthy subjects. Our results were supported by a comparison of the sequenced genomes of isolates from the same microbiota samples and a meta-analysis of published gut metagenomes.

Conclusions: The genomic diversity of Crohn's disease-associated E. coli within and among the patients paves the way towards an understanding of the microbial mechanisms underlying the onset and progression of the Crohn's disease and the development of new strategies for the prevention and treatment of this disease.

Keywords: Gut microbiota, Crohn's disease, Escherichia coli, Gene content, Inflammatory bowel diseases, Metagenomics, Pangenome

\footnotetext{
* Correspondence: a.tyakht@gmail.com

${ }^{1}$ Federal Research and Clinical Centre of Physical-Chemical Medicine, Malaya

Pirogovskaya 1a, Moscow 119435, Russia

${ }^{2}$ Moscow Institute of Physics and Technology, 9 Institutskiy per.,

Dolgoprudny, Moscow Region, Russian Federation141700

Full list of author information is available at the end of the article
}

(c) The Author(s). 2018 Open Access This article is distributed under the terms of the Creative Commons Attribution 4.0 International License (http://creativecommons.org/licenses/by/4.0/), which permits unrestricted use, distribution, and reproduction in any medium, provided you give appropriate credit to the original author(s) and the source, provide a link to the Creative Commons license, and indicate if changes were made. The Creative Commons Public Domain Dedication waiver (http://creativecommons.org/publicdomain/zero/1.0/) applies to the data made available in this article, unless otherwise stated. 


\section{Background}

Gut microbiota disequilibrium is among the accepted hallmarks of Crohn's disease (CD). Although the aetiology of this disease is still not completely clear, in addition to genetics and lifestyle, the microbial community structure is among the factors contributing to its pathology [1]. The importance of understanding the role of microbiota in CD and the promising opportunity of the therapeutic modulation of its composition have been recently demonstrated by successful $C D$ treatment via faecal transplantation in pilot studies (for example, in adult [2] and paediatric [3] patients; a recent meta-analysis includes 11 studies [4]).

Using metagenomic high-throughput sequencing, the changes in the structure and function of gut microbiota in CD can be assessed. Surveys of $16 \mathrm{~S}$ rRNA and "shotgun" formats have revealed a decrease in the total species diversity and abundance of butyrate-producing species accompanied by the growth of Proteobacteria and other opportunist species [5-8]. An analysis of "shotgun" sequencing data has provided evidence of shifts in the functional composition, including the increased presence of genes linked to inflammation, oxidative stress (including glutathione transport) and amino acid degradation [5]. Changes in microbial metabolism in the ileum of CD patients have also been reported based on proteomic studies [9]. Correlation analyses of microbial gene abundance levels have shown that the decrease in community richness in $\mathrm{CD}$ is likely due to the extinction of understudied species without representative reference genomes [10]. In addition to the changes in the microbial community, the gut virome composition has been found to be abnormal in patients with CD $[11,12]$.

The metagenomic profiling of microbiota in patients with $\mathrm{CD}$ is complicated by certain methodological challenges. Antimicrobial, anti-inflammatory and other types of treatments represent independent factors that strongly influence the community composition $[7,13]$. To distinguish the effect of medical prescriptions from the effect caused by the disease, the metagenome has been examined in treatment-naive patients, e.g., children [14]. However, in the above-mentioned study, the ileal microbiota was profiled using $16 \mathrm{~S}$ rRNA sequencing of biopsy samples, which limited the assessment of the gene content (biopsy samples are not directly applicable due to the absolute dominance of human DNA over the microbial fraction [15]). Furthermore, species with highly similar $16 \mathrm{~S}$ rRNA gene sequences might carry substantially different sets of accessory genes, including groups of genes with special clinical significance, such as antibiotic resistance determinants and virulence factors [16].

To date, no single microbial species has been identified as an unambiguously strong factor contributing to the onset of Crohn's disease. However, Escherichia coli, particularly the adherent-invasive pathotype (AIEC), is among the species often detected in increased abundance in CD patients $[17,18]$. This species is a beneficiary of inflammation that is able to persist in the gut mucosa in inflammatory tissue. Specific varieties isolated from CD patients with the AIEC pathotype are able to invade epithelial cells, survive in macrophages $[19,20]$ and form biofilms [21]. The increase in Proteobacteria in the ileum has been shown to be correlated with the Pediatric Crohn's Disease Activity Index (PCDAI) [22]. Physiologically, the survival of $E$. coli is linked to an increase in the reactive oxygen species levels and a decline in the populations of commensal bacteria which normally provide a protective effect in the host's intestine. E. coli is not associated with the underlying pathology of $\mathrm{CD}$ but rather a marker of the inflammatory process [23]. Although E. coli is among the most examined model organisms, the enormous genomic variability of this species poses many questions regarding its functioning in vivo. Its identity and role in CD are widely discussed. In $\mathrm{CD}$, are the $E$. coli present clonal or are there distinct genotypes of the organism present? What are the specific features distinguishing $E$. coli in $\mathrm{CD}$ patients from $E$. coli in healthy subjects? Finally, is the species a primary cause of Crohn's disease contributing to the onset of the disease via interplay with the other risk factors or a secondary "first available" species emerging in an environment of general gut imbalance that exacerbates disease progression?

Here, we attempted to decipher the microbial causal factors of $C D$ at multiple levels, i.e., from the gut community level to the single species level (E. coli), by integrating cultivation-independent and -dependent methods. We conducted a "shotgun" metagenomic analysis of gut microbiota in CD using stool and ileal content samples collected from patients with $\mathrm{CD}$ at two clinical centres.

\section{Results and discussion \\ Microbiota in Crohn's disease patients exhibits a range of abnormal community structures}

Generally, stool and ileal metagenomes from the same CD patients tended to be related by taxonomic composition as follows: while the dissimilarity between the paired stool and ileal samples from the same subject was higher than between the replicates of the same sample using different platforms (Bray-Curtis dissimilarity 0.52 \pm 0.30 vs. $0.22 \pm 0.03$, SOLiD vs. Ion Torrent, one-tailed Welch t-test $p=0.04)$, it was significantly lower than the between-pairs variation $(0.82 \pm 0.15, p=0.05)$. The species-level profiling of the microbiota showed pronounced dysbiosis as a prevalence of various abnormal community types driven by opportunistic pathogens (Fig. 1). 


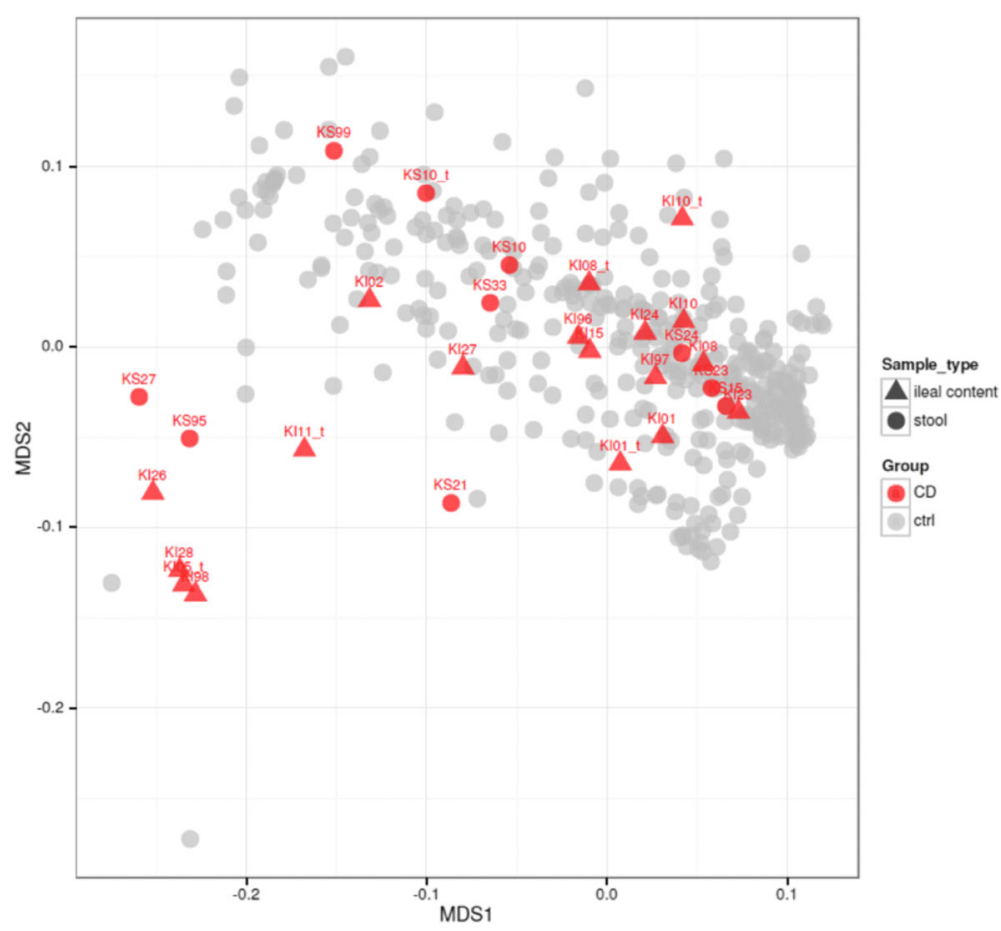

Fig. 1 Variation of species-level composition in healthy controls depends on the sample type. Multi-dimensional scaling plot using the wholegenome UniFrac metric. Each point corresponds to a single metagenome; sample type is shown by the shape, while the color shows whether the sample was collected from a CD patient or a subject from an external control group. The external control group included 385 stool metagenomes from healthy Russian, American, Danish and Chinese populations

The comparison of the stool metagenome compositions with those of a healthy Russian population as an external control group $(n=96)$ [24] showed a significant shift in the levels of many bacterial species, including a marked enrichment in opportunistic pathogens and decrease in commensal flora (see Fig. 2; a complete list of the significantly over- and underrepresented species is provided in Additional file 1: Table S1). Moreover, the CD metagenomes are dominated by pathogens that were undetected or had a relative abundance close to zero in most healthy subjects (the central observations shown in Fig. 2 are listed below). There is an abnormal domination of Proteobacteria (Escherichia, Enterobacter, Pseudomonas and Klebsiella). The major drivers included Enterococcus faecalis, faecium and casseliflavus, which are pathobionts implicated in nosocomial infections that are able to survive oxidative stress and are associated with inflammation $[25,26]$. The observed dominance of the species listed is consistent with existing data regarding the CD gut microbiota $[7,14,22]$. In one of the ileal samples, we identified an unexpected presence of Aeromonas hydrophila and veronii (5\%; see Fig. 2); infection with this enteropathogen is associated with a poor clinical outcome in IBD [27]. In another patient, both the stool and ileal samples contained a high fraction (0.4 and 4.2\%) of Fusobacterium varium, which is a species associated with and able to induce ulcerative colitis [28]. In one ileal sample and one stool sample, we detected a significant fraction of Clostridium difficile (3.2 and 7.2\%, respectively). Other opportunist pathogens overrepresented in some CD metagenomes included Clostridium nexile and Clostridium clostridioforme [29]; Veillonella and Flavonifractor; the sulfate-reducing bacterium Bilophila wadsworthia, which is a pathogen associated with appendicitis and other intra-dominal inflammatory disorders [30]; and Streptococcus infantarius, which is associated with colon cancer [31]. Some of the CD were enriched in Ruminococcus gnavus, which is among the few known representatives of the genus that consumes mucosa and is linked to IBD [32]. The increased levels of Lactobacillus rhamnosus (in both stool and ileal samples from one patient, 53 and $8 \%$ ) and Bifidobacterium breve (1 stool sample, $4 \%$ ) were likely due to the intake of probiotic or dairy products; however, certain studies have shown an increase in the respective genera in microbiota from IBD patients [33, 34] and, for B. breve, from patients with alcohol-induced liver cirrhosis [35]. Notably, as seen from Fig. 2, CD metagenomes with increased levels of Bacteroides were more prevalent than 


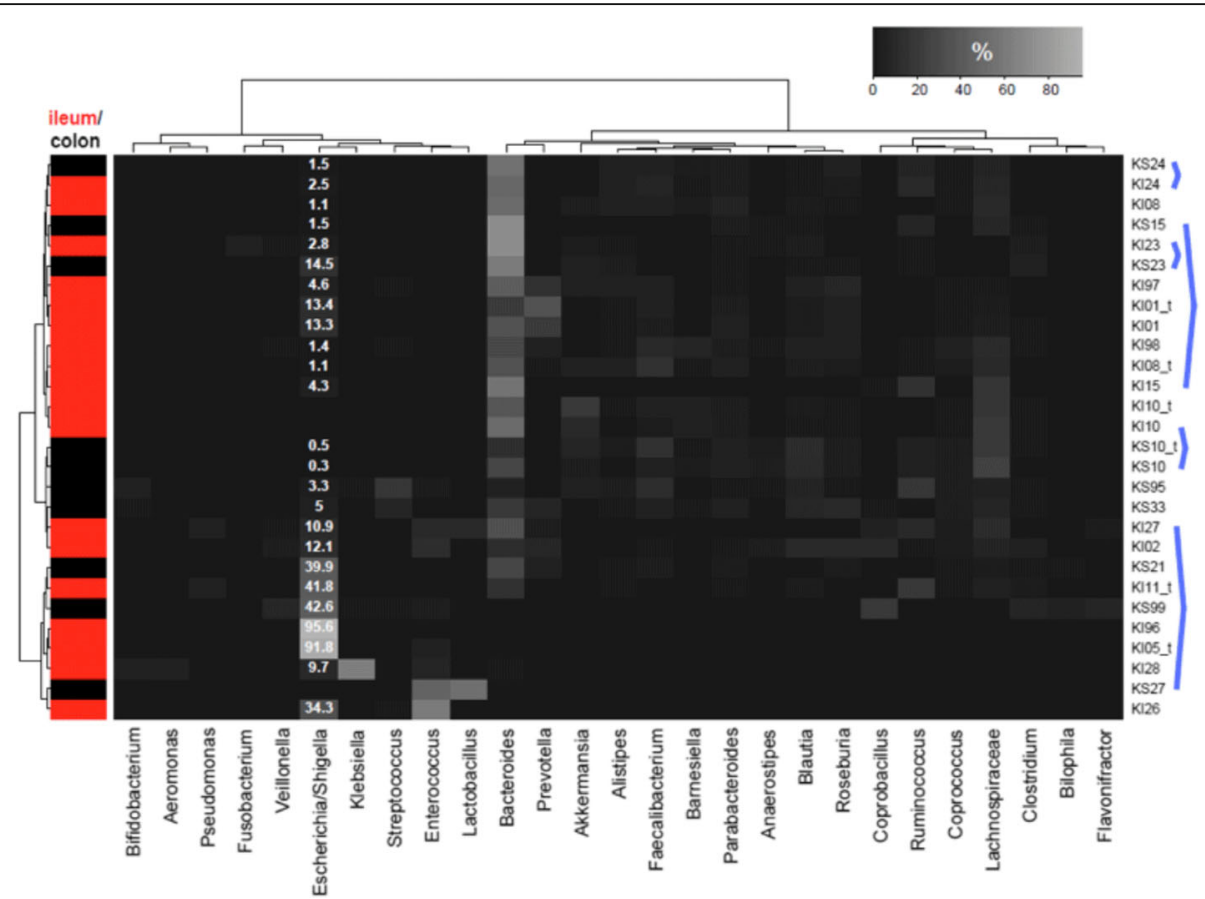

Fig. 2 Taxonomic composition of gut metagenomes in Crohn's disease patients is characterized by the pronounced presence of Escherichial Shigella. The heatmap shows relative abundance of microbial genera (columns) in microbiota samples (rows). The genus levels are provided in percentages of the total bacterial abundance. The blue lines connect pairs of stool and ileal metagenomes from the same patients. Hierarchical clustering is performed using whole-genome UniFrac metric for rows and (1 - Spearman correlation) - for columns; linkage was performed by Ward's method. Only the major genera ( $>3 \%$ of the total abundance in at least one sample) are shown

those with Prevotella (13 vs. 1 metagenomes with a genus abundance $>30 \%$ ). Levels of Faecalibacterium prausnitzii were also significantly decreased compared with that in the healthy Russian population $(4.0 \% \pm 5.1 \%$ vs. $8.5 \% \pm 8.5 \%$, Mann-Whitney test, adjusted $p=0.042$ ). This microorganism is recognized as an inhibitor of inflammation in the gastrointestinal tract [36, 37]. Multiple species from Roseburia, Coprococcus, Eubacterium and other related genera from the Firmicutes phylum known to be important gut butyrate-producers were also significantly decreased (Fig. 2).

\section{Metagenomic analysis of $E$. coli genome suggests the existence of multiple strains within the same patients} One of the most obvious differences between the CD patients and the healthy populations from Russia and other countries was the order of magnitude increase in the Escherichia coli relative abundance in the stool samples $(2.4 \% \pm 16.5 \%$ vs. $0.2 \% \pm 7.7 \%$, one-tailed Mann-Whitney test $p=0.00167)$. The ileal E. coli levels were also high $(2.8 \% \pm 4.1 \%)$ but were not correlated with the respective stool levels in the same patients $(n=5$, Pearson correlation $r=-0.22)$. Previously, we performed a cultivation-dependent study of this species associated with $\mathrm{CD}$ via the isolation and genome sequencing of a subset of the same microbiota samples [38]. To deepen our understanding of the specifics of CD-associated E. coli, here, we compared the gene content of this bacterial species reconstructed from "shotgun" metagenomic data with genomes isolated from gut microbiota samples from the same patients (Additional file 1: Table S2).

Overall, the analysis of the metagenomes confirmed the presence of the species with genomes highly similar to that of the respective isolates. The mapping of the metagenomic reads to the corresponding E. coli isolate genomes and analysis of the read coverage using the maximum expected coverage (MEC) algorithm (see Materials and methods) showed that for each of the seven metagenomes analyzed, the corresponding $E$. coli isolate genome was the most similar and that the similarity was significantly higher than the similarity between random "genome-metagenome" pairs (MEC $=0.99 \pm 0.01$ vs. $0.81 \pm 0.08$, one-tailed Mann-Whitney test, $p \leq 10^{-7}$ ) (Additional file 1: Figure S1). 
The metagenomic data also supported certain important extra-chromosomal specifics of $E$. coli identified via the genomic analysis of the isolates. The mapping of the metagenomic reads to the sequence of the plasmid pLF82 (present in the CD-associated E. coli strain LF82 [23]) yielded 4 samples with a high coverage $(17-73 \%$ of the plasmid sequence was covered), and all four of the corresponding $E$. coli isolate genomes were shown to have sequences with a high level of identity to pLF82 [38] (Additional file 1: Table S3A). Moreover, some subjects from the healthy Russian population also had metagenomic sequences with identity to this plasmid (Additional file 1: Table S3B); interestingly, these samples had increased fractions of E. coli and other opportunistic bacteria (Streptococcus, Enterococcus and Klebsiella).

For a more precise comparison of the genomes and metagenomic profiles of $E$. coli from the same samples, we performed an analysis of metagenomic single-nucleotide polymorphisms (mSNPs) (see Materials and methods). In total, there were 5 "genomemetagenome" pairs in which the metagenome produced sufficient coverage of the $E$. coli genome to perform this analysis. In 3 of these pairs, the distance was orders of magnitude lower than the distance within the mixed "genome-metagenome" pairs (distance $\mathrm{d}=0.00026-0.00520$ vs. $0.018 \pm 0.011, p=0.002$, one-tailed Mann-Whitney test; see Additional file 1: Table S4). However, in the other 2 pairs, the distance was significantly higher $(\mathrm{d}=0.016$ and 0.010$)$. To examine the possibility of subspecies-level diversity in the discrepant pairs, we estimated the mSNP allele frequency. Interestingly, in each of these two pairs, the fraction of mSNPs with a second major allele identical to the letter present in the respective genome was higher than that of any of the mixed "genome-metagenome" pairs involving the same genome $(95 \%$ vs. $84 \% \pm 4$ and $75 \%$ vs. $62 \% \pm 5 \%$, respectively). Our results suggest the existence of more than one $E$. coli strain in the microbiota samples and that the sequenced genome corresponds to one of the dominant strains. The analysis of the subspecies-level diversity of the metagenomes using an alternative approach, i.e., the ConStrains tool [39], also revealed the presence of two or more strains of E. coli in 7 of the 28 analysed metagenomes (see Additional file 2: Table S5).

\section{CD-associated $E$. coli is genetically diverse}

To assess the gene composition landscape of $E$. coli as reflected by the metagenomic data, we performed pre-mapping to a global gut microbial gene catalogue [40] and selected the genes belonging to E. coli from the catalogue. Initially, the E. coli pangenome was identified using a representative set of various $E$. coli genomes, including commensal, pathogenic and other strains; then, based on the sequence similarity, the genes corresponding to the $E$. coli pangenome were extracted from the gene catalogue (see Materials and methods). The analysis of the presence/absence of genes was performed on the level of orthology groups (OGs). We separately analysed the part of the pangenome corresponding to an accessory genome (AG) containing 2993 of the 5598 OGs.

Our previous genomic analysis of strains isolated from gut microbiota of $\mathrm{CD}$ patients showed that the functions that mostly differentiate $\mathrm{CD}$-associated $E$. coli from commensal strains include the utilization of propanediol (and other sugar alcohols) and iron uptake [38]. We decided to explore how this understanding conforms to the results obtained from the "shotgun" metagenomic profiling of the same and an additional set of samples from $\mathrm{CD}$ patients. To analyse the metagenomic profile of the $E$. coli gene content in the context of the available genomic data, we converted a representative set of $E$. coli genomes to an accessory genome presence/absence profile (see Materials and methods) and compared this profile to metagenomic E. coli images of $\mathrm{CD}$ patients from Russia, the USA [14] and Denmark [10] and healthy populations worldwide. Using the AG profiles, the metagenomic $E$. coli images appeared to be more similar to the genomes of the strains isolated from the same samples $(0.25 \pm 0.09, n=9$ comparisons, binary metric) than all unrelated AG profiles of the worldwide populations were to each other $(0.37 \pm 0.09$, one-tailed Welch t-test, $p=0.001)$; the same result was observed when the comparison was performed using only the $E$. coli virulence genes (17 genes selected as described in the Methods; $0.20 \pm 0.28$ vs. $0.5 \pm 0.19$, respectively; $p=0.04082$ ). The paired AG profiles of the stool and ileal samples from the same patients were highly similar $(\mathrm{d}=0.06$ and 0.17 , $n=2$ pairs).

Interestingly, the hierarchical cluster analysis of the AG profiles showed a high level of genetic diversity of E. coli in the CD metagenomes (Fig. 3) suggesting that $\mathrm{CD}$-associated $E$. coli are not a homogenous group but rather consist of multiple genotypes with diverse genomic repertoires (clustering by the above-mentioned 17 virulence genes also showed diversity, see Additional file 1: Figure S2). The distribution of the dissimilarity values among the AG profiles was comparable to that of the worldwide AG profiles $(0.39 \pm 0.11, p=0.16$; one sample per patient). A similar effect was observed in the Spanish CD patients $(0.35 \pm 0.06)$. The USA treatment-naive patients tended 


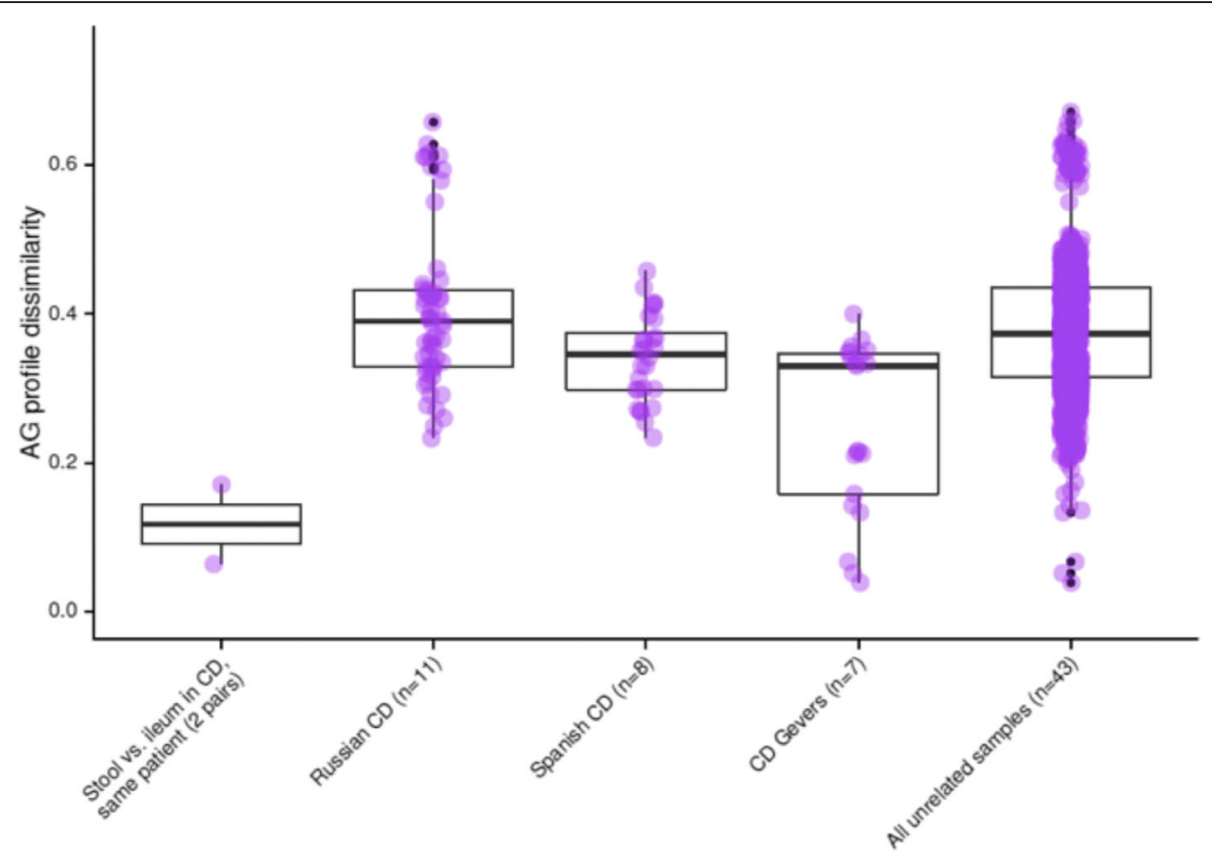

Fig. 3 Variability of E. coli accessory gene presence profile across different groups of metagenomes. The boxplots show the distributions of pairwise dissimilarity of accessory genome (AG) profiles of E. coli calculated for all possible pairs from the following groups of samples: stool and ileal samples from the same Russian CD patient; all Russian CD patients; Spanish CD patients; USA treatment-naive CD patients; as well as the pairs between all unrelated samples. The scatterplots reflect the same information as the boxplots in a more detailed way

to be more similar in their AG profiles $(0.33 \pm 0.12)$, which was even more pronounced after removing the single distant sample SAMN02674793 (0.21 \pm 0.11$)$.

Except for several strongly outlying samples (CD metagenomes from the same patient KS23 and KI23 with their respective genome RCE03 and three Chinese metagenomes), the tree contained 2 main branches (Clades 1 and 2; see Fig. 4). Regarding the metagenomes from the Russian CD patients, the distribution highly conformed to the results obtained using the multiple alignment of the E. coli whole genomes [38]. Clade 1 (including 16 metagenomes and 20 genomes) included all 4 major known CD-associated E. coli strains (LF82, O83:H1 [41], UM146 [42] and HM605 [43]) and several other pathogenic strains included in the O6 serogroup. Remarkably, among all CD metagenomes included in the tree, Clade 1 included only 2 of the 13 Russian and only 1 of the 13 Spanish CD metagenomes; however, almost all (6 of 7) of the metagenomes from the treatment-naive USA patients were located in Clade 1.

To identify the genotype of the most recent common ancestor of the pathogen-enriched Clade 1, we identified the genes prevalent in this clade $(n=1128$; OGs detected in $>80 \%$ of the metagenomes) and at the same time rare in Clade $2(n=798$; OGs detected in $<20 \%$ of the metagenomes). Forty-seven OGs were obtained. Furthermore, we refined the list by excluding the OGs with a similar OG detected in most Clade 2 members (see Materials and methods), ultimately yielding 34 genes.

The list representing the signature of Clade 1 (Additional file 1: Table S6) contains several genes that are remarkable from the pathobiont arsenal perspective. The proteins coded by some of these genes are known to play important roles in biofilm formation as follows: holin-like protein CidA facilitates the release of DNA that fortifies the biofilm [44], and toxin-antitoxin biofilm protein TabA influences biofilm dispersal [45]. Putative neuraminidase (sialidase) is another gene involved in mucus degradation that potentially contributes to the mucus adherence of $E$. coli as follows: sialidases are known factors involved in the pathogenesis of microbial infections by helping bacteria survive in the outer mucosa layer by cleaving mucin glycoproteins $[46,47]$. The levels of its particular type, intramolecular trans-sialidase, have been shown to increase in microbiota of IBD patients [48]. Several genes are associated with cell adhesion and fimbriae facilitating successful colonization of the gut, including long polar fimbrial protein LpfD and putative fimbrial-like protein YcbV precursor. The presence of the metallo-beta-lactamase superfamily proteins suggests that resistance to clinically important antibiotics is prevalent among the members of Clade 1. Finally, the presence of microcin-E7 immunity protein 


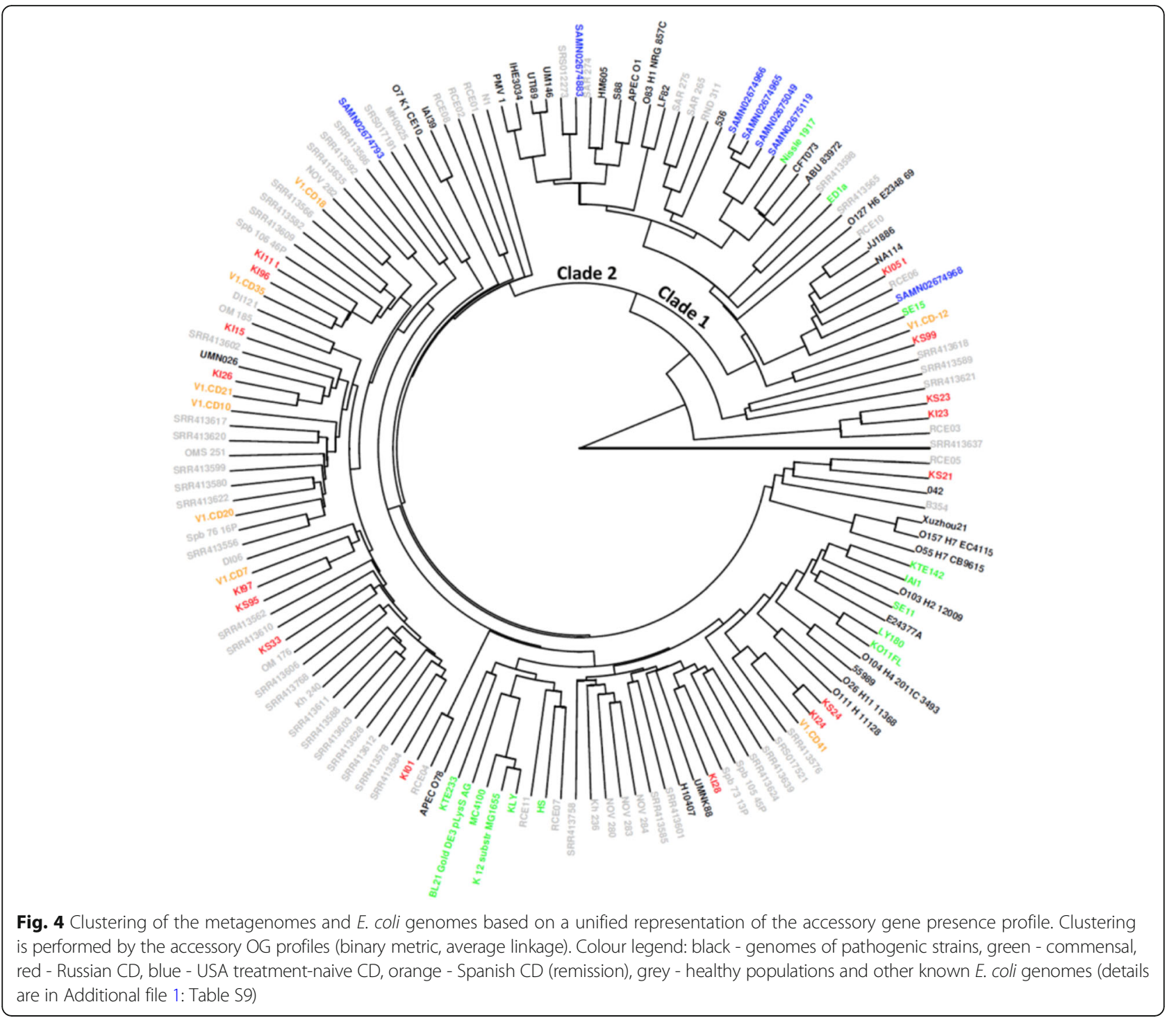

(conferring immunity to toxin colicin E7 produced by bacteria) suggests that such a toxin that is normally carried on a plasmid is also prevalent in CD-associated $E$. coli belonging to this clade.

For further validation, a cluster analysis of the reconstructed E. coli gene content was additionally performed using the gene-family profiles obtained using an alternative approach, i.e., PanPhlAn [49]. Once the samples from the Russian CD patients were clustered, a tree visually similar to that produced using our approach was obtained (Additional file 1: Figure S3A); this similarity was confirmed by comparing the distance matrices yielded using our method and PanPhlAn (Mantel test $R$ $=0.928, p=0.001$ ). Once this set was extended (as an example) by 6 metagenomes from USA treatment-naive $\mathrm{CD}$ patients and 7 metagenomes from the Chinese control group, the resulting tree also included 2 main branches that were generally similar to those in the tree based on the AG profiles (Additional file 1: Figure S3B; Mantel test $R=0.8824, p=0.001$ ).

\section{Comparison of accessory genomes to matched controls} shows the lack of genetic commonalities in CD-associated E. coli

Remarkably, most Russian CD metagenomes were located in Clade 2 (36 genomes and 68 metagenomes; see Fig. 4). Clade 2 contains few pathogenic (or disease-associated) strains. Therefore, we analysed the contents of Clade 2 in another way. E. coli in each particular case of CD can possess a specific set of genes that allow it to thrive in the gut environment and produce inflammation. To examine this question in greater detail, we compared the AG profile of each CD metagenome in Clade 2 (11 Russian and 8 non-Russian patients) with the closest match to the metagenome of a healthy subject. While most subjects were 
not examined endoscopically, at the time of sampling, the subjects did not manifest signs of intestinal discomfort.

Nineteen matched control comparisons were performed. The matched controls closest to $\mathrm{CD}$ in terms of the AG profile were the healthy Russian ( $n=10$ comparisons) and Chinese $(n=9)$ populations; the samples within the compared pairs were 1.4 times closer than random pairs $(0.23 \pm 0.03$ vs. $0.33 \pm 0.05, p=2.268 \mathrm{E}-14)$. In each comparison, $227 \pm 76$ genes per comparison were identified to be $\mathrm{CD}$-specific (present in $\mathrm{CD}$ but lacking in the non-CD control; 1704 unique genes in all comparisons in total). This distribution was found to be generally comparable with that obtained in the inverse comparison $(261 \pm 165$ genes per comparison present in the E. coli profile of a control but not in that of a matching $C D$ patient; paired Mann-Whitney test $p=0.52 ; n=1763$ unique genes in total).

We analysed the genes that are commonly present in E. coli in $\mathrm{CD}$ patients but lacking from one of the matched controls (identified in $\geq 5$ of the 19 comparisons). In total, 175 and 205 genes were identified in the $\mathrm{CD}$ and control metagenomes, respectively (80 and 137 after discarding the genes with unknown functions, see Additional file 1: Table S7). The lists of $\mathrm{CD}$ - and control-specific genes contained a similar fraction of genes related to bacteriophages $(15 \%$ vs. $19 \%)$ and horizontal gene transfer $(2.5 \%$ vs. $3.6 \%)$. No genes related to biofilm formation were included in the lists. However, the CD-specific list was enriched in mercury metabolism genes ( $5 \%$ vs $0 \%$ ). The $E$. coli accessory genome diversity results showed that most $\mathrm{CD}$ metagenomes harbouring E. coli strains are genomically diverse and distant from the AIEC pathotype typically described as CD-associated (contained in Clade 1).

Overall, the increasing incidence of inflammatory bowel diseases, including Crohn's disease, in developed countries is a frightening prospect in terms of morbidity and a challenge for the modern global healthcare system. A meta-analysis showed that the annual incidence is as high as 12.7 and 20.2 per 100,000 person-years in Europe and North America, respectively [50]. The Western lifestyle includes not only the acquisition of material wealth and social security but also the following undesirable additions: underexposure to bacteria during birth and at an early age, the consumption of industrial foods rich in preservatives and artificial additives, a sedentary lifestyle, and decreased intestinal motility. A plethora of these previously underestimated factors contributes to the imbalance of the "host-microbiota" system and is associated with an increased risk of IBD. Given the rapid globalization, Crohn's disease is a potential threat for all individuals.

To date, studies attempting to identify the ultimate causative factors of this disease have not been successful.
In particular, although microbiota may be involved in the pathogenesis [51], its participation is not completely elucidated. The available results suggest that no single reference healthy gut microbiota exists [52]. The species-level composition is known to vary widely but still provides functional homeostasis [53]. We suggest that following external perturbations, the emerging pathological process might easily resolve in some subjects, but in other subjects this process may transform into chronic inflammation and may be due to an imbalance in the microbiota [54]. An important question is where to search for markers of this imbalance, i.e., at the global community, subcommunities or individual opportunist pathogen levels. Several studies have shown associations between particular gut bacterial species and Crohn's disease. Some of these species have been hypothesized to be causative agents of the disease $[17,55]$. We approached this problem from different scales of the gut system, i.e., at the single species level, particularly $E$. coli, which is among the most referenced species in this context [56-58], and the whole gut community level.

Although E. coli is the first and most well-studied human gut species, its levels are normally relatively low compared with species that are members of the dominant phyla (Bacteroidetes, Firmicutes and Actinobacteria). The low levels are likely linked to the fact that $E$. coli has a specific ecological niche as follows: this species does not metabolize polysaccharides [59], which is the main source of energy in the colon. While Proteobacteria may normally prevail in the neonate gut to prepare the niche for the further colonization of commensals [58], in adults, the increased abundance of E. coli is associated with various diseases, including colon cancer [60], liver diseases [61], cystic fibrosis [62], and alcoholic dependence syndrome [35] probably due to the inflammation that supports the growth of $E$. coli via the selective suppression of commensal taxa [63]; this increase can also occur following treatment with proton pump inhibitors [64].

In our analysis of the gut communities in patients with Crohn's disease, we revealed significant dysbiosis in stool and ileal metagenomes. One of the most abundant genera was Bacteroides. The increase of Bacteroides has been associated with the risk for the development of intestinal inflammation [65]. In general, among all commensals, Bacteroides fragilis had the highest abundance. Enterotoxigenic $B$. fragilis which possess the $b f t$ gene has been hypothesized to be associated with IBD [66]; however, the two $b f t$ gene sequences from the gene catalogue were not detected in any of the CD metagenomes (data not shown). The observed increase in opportunistic pathogens is consistent with previously published results $[8,14]$. Interestingly, the disruption of the community structure appears to be due to an increase in 
different opportunist species in each patient and, thus, lacks a single direction. However, the significantly increased fraction of E. coli in comparison with that in the healthy populations from Russia and other countries was a universal feature. The identification of the specific gene content of $\mathrm{CD}$-associated $E$. coli could enhance our understanding of the gene determinants of the active role of the species in inflammation and, based on variability, differentiate possible scenarios related to pathogenesis. We previously demonstrated the broad variability of $\mathrm{CD}$-associated $E$. coli using genomic sequencing of isolates of gut microbiota from patients [38]. Here, we extended the scope of the analysis by approximating the consensus composition of the accessory genome of the species using metagenomic data. Our approach allowed us to avoid cultivation-associated biases and include the extensive volume of published human metagenomic datasets in a comparative analysis.

CD-associated $E$. coli has been previously suggested to be predominantly represented by AIEC strains, including LF82 and O83:H1 [67]. However, our results show that a large number of $E$. coli genotypes were detected in $C D$ patient metagenomes. Genotypes similar to those of the AIEC strains were only detected in a small fraction of $\mathrm{CD}$ patients from the Russian and other populations worldwide. The respective Clade $1 \mathrm{E}$. coli strains from $\mathrm{CD}$ patient metagenomes possessed genes associated with biofilm formation, antibiotic resistance, mucus and cell adhesion. The presence of genes associated with virulence does not unambiguously imply the pathogenicity of a strain $[68,69]$. Thus, a pathogenicity score based on the number of such genes in a genome is unreasonable. However, the genes identified can be considered genetic determinants conferring a high level of fitness to $\mathrm{CD}$-associated strains in the human gut (based on their high abundance). This hypothesis is supported by the fact that in addition to AIEC and several other pathogenic strains, the probiotic strain Nissle 1917 is included in Clade 1. Most CD-associated E. coli were found to be located in the Clade 2. An alternative approach for identifying genetic commonalities among $E$. coli in this clade, i.e., comparing the AG profiles with those of matched healthy subjects, only yielded an enrichment in mercury metabolism genes. As heavy metal resistance genes are often carried on plasmids along with antibiotic resistance and virulence factors $[70,71]$, we speculate that their prevalence is linked to the higher prevalence of mobile elements (particularly plasmids) in CD-associated E. coli than in E. coli inhabiting the gut of healthy subjects. Interestingly, the richness of the gene repertoire in $\mathrm{CD}$-associated $E$. coli was comparable to those that are in non-CD subjects, although it is generally accepted that pathogenic strains of $E$. coli tend to possess larger genomes than commensal strains [72].
The lack of specific virulence-associated genes commonly distinguishing CD-associated $E$. coli in Clade 2 from the matched control E. coli suggests that there are no universal genetic determinants conferring Clade $2 \mathrm{E}$. coli virulence in CD. Generally, our observations suggest that CD-associated E. coli do not possess a specifically defined gene composition but is represented by a broad range of biotypes varying in gene content; the species is likely involved in disease progression rather than onset. These results are consistent with a recent cultivation-based study investigating AIEC [73] that failed to identify a molecular property exclusive to this phenotype. Our conclusions support the concept that Crohn's disease is a syndrome, i.e., a disease in which similar manifestations in multiple cases are caused by different factors in each case. It is possible that some genes detected in our prediction of the $E$. coli accessory gene content from the metagenomic data in fact originated from other species (i.e., had been obtained from Escherichia/Shigella spp. via horizontal transfer). However, the general validity of our method of assessing the $E$. coli accessory genome content from the metagenome is supported by the high similarity with the core genomic data of the isolates from the same microbiota samples.

Furthermore, our results extended the previous findings as follows: at a subtler level, the analysis of the E. coli subspecies-level diversity within a microbiota of $C D$ patients performed using our metagenomic SNP profiling algorithm and the ConStrains algorithm revealed that some CD patients host multiple strains of $E$. coli in their gut microbiota. Although the data was derived from only a few samples, the considerable size of the effect is supported by the fact that in each of the samples, we identified thousands of sufficiently covered mixed-type mSNPs supporting the heterogeneity. The effect should be further examined via the extensive isolation of multiple strains using a variety of growth media and genome sequencing. Such diversity might reflect microevolutionary snapshots of $E$. coli or its co-existence at different loci in the gut. Previous genomic analyses of isolates demonstrated the possibility that multiple strains of the same species co-exist within the microbiota of the same subjects, such as, Helicobacter pylori in the stomach [74, 75] and Propionibacterium acnes on the skin [76]. The metagenomics data also provided evidence for such a phenomenon in the microbiota of healthy subjects [39]. Here, for the first time, we demonstrated the genomic heterogeneity of $E$. coli within the same CD patients. This understanding is eye-opening for clinicians because it implies the need to carefully revise the treatment for Crohn's disease as follows: an antimicrobial therapy scheme targeted against one biotype of $E$. coli might be ineffective against the other biotypes associated with the condition and may cause collateral damage to both the commensal microbiota and 
organism of a patient. In particular, there is a need to use a diverse set of organisms as part of probiotic treatment scheme as the experimental evidence shows that multiple E. coli pathotypes occupy significantly different ecological niches [77].

\section{Conclusions}

Our results expand the current understanding of how microbial components contribute to Crohn's disease onset and progression and pave the way for the development of better strategies for personalized diagnostics and treatment of inflammatory bowel diseases.

\section{Methods \\ Sample collection}

The samples were collected from a cohort of patients with CD $(n=19)$ enrolled at two clinical centres (Moscow Clinical Scientific Center and State Scientific Center of Coloproctology, Moscow, Russian Federation). The inclusion criteria were as follows: patients aged 18 years or older who were endoscopically and radiologically diagnosed with and histologically confirmed to have Crohn's disease. The exclusion criteria included signs of indeterminate colitis, infectious diseases, anamnesis of total colectomy, the presence of stoma, and antibiotic treatment. The faecal samples were collected prior to the preparation for endoscopy. The bowel preparation was performed with a polyethylene glycol solution. The patients underwent ileocolonoscopy at the clinical centres. During this procedure, the ileum liquid content was aspirated. In total, 9 stool and 15 ileal samples were obtained (Additional file 1: Table S8).

\section{Sample preparation protocol}

The stool samples were prepared according to the following protocol. A portion of $0.3-0.5 \mathrm{~g}$ of faeces was added to $10 \mathrm{ml}$ of PBS, vigorously vortexed for $2-5 \mathrm{~min}$ and repeatedly cooled on ice. Human cells and debris were pelleted by centrifugation for $10 \mathrm{~min}$ at $0.1 \mathrm{~g}$. The supernatants were collected and placed on ice. The resuspension and centrifugation of the pellet were repeated 3-5 times. The combined supernatant was centrifuged for $20 \mathrm{~min}$ at $13.5 \mathrm{~g}$ to collect the microbial fraction. The pellet was resuspended, washed twice with PBS and stored at $-20^{\circ} \mathrm{C}$.

The ileal samples were collected as ileal lavage fluid collected during endoscopy by washing the ileal wall with saline. In total, $1 \mathrm{ml}$ of fluid was used for the DNA extraction from such samples. Unlike the stool sample preparation, a lower dilution (3-5 times) and a lower number of repeats of resuspension and centrifugation (no more than twice) were performed.

\section{Preparation of metagenomic libraries and "shotgun" sequencing}

The "shotgun" libraries were prepared for the sequencing platforms SOLiD 4, SOLiD 5500 and Ion Torrent (Life Technologies, USA). The sequencing was performed according to the manufacturer's instructions. For SOLiD 4, the SOLiD Fragment Library Construction Kit, SOLiD Fragment Library Barcoding Module 1-16, SOLiD EZ Bead TM E80 System Consumables, SOLiD ToP Sequencing Kit and MM50/5 (Life Technologies, USA) were used. For SOLiD 5500, the 5500 SOLiD Fragment Library Core Kit, SOLiD Fragment Library Barcoding Kit, SOLiD FlowChip Kit, SOLiD FWD SR S50 Kit and SOLiD Run Cycle Buffer Kit (Life Technologies, USA) were used. For Ion Torrent PGM, the Ion Xpress Plus Fragment Library Kit, Ion Sequencing Kit, Ion PGM Template OT2 200 Kit, Ion PGM Sequencing 200 Kit and Ion 318 Chip Kit (Life Technologies, USA) were used.

Three of the samples were sequenced using more than one platform; in total, 28 metagenomic read sets were obtained (Additional file 1: Table S8).

\section{Taxonomic and functional profiling of metagenomes}

All analytical steps of the study are summarized in the Additional file 1: Figure S4. The taxonomic composition analysis was performed by mapping the metagenomic reads against a representative non-redundant reference catalogue of 353 gut microbial genomes [78] and estimating the relative abundance levels based on the obtained coverage profiles as previously described [79]. The functional composition was assessed in a similar way as previously described by using a reference gene catalogue containing $3.3 \mathrm{mln}$ gut microbial genes [40]. For the comparative analysis, stool metagenomic datasets from 3 studies involving the following healthy populations worldwide were used: China $(n=68)$ [80], Denmark $(n=85)$ [40], and USA $(n=138)$ [81]; in addition, Crohn's disease patients from the USA (treatment-naive paediatric patients; $n=17$ ) [14] and Spain (clinical remission, $\mathrm{n}=13)[10,40]$ were included.

\section{Analysis of maximum-expected coverage}

The bacterial species present in a microbiota sample might possess genome(s) that considerably differ from the reference genome available as a representative of this species. In such cases, even if the sequencing depth was infinitely increased, the covered part of the genome could converge to a value less than $100 \%$; the higher the value, the more representative the reference genome. To assess this limit, we assumed that the non-covered part of the genome exponentially decreases as the coverage depth increases and introduced a maximum expected coverage (MEC) value as follows: 


$$
\alpha=\frac{f}{1-\exp \left(-\frac{\mathrm{NL}}{G}\right)}
$$

where $N$ is the number of mapped reads, $L$ is the read length, $G$ is the genome size, and $f$ is the number of positions in a genome that were covered by at least 1 read. The MEC is an indirect measure of the similarity of the gene content between the reference genome and the bacterial species present in the analysed sample.

\section{Analysis of metagenomic SNPs}

To assess the subspecies level genomic variability of $E$. coli using "shotgun" metagenomes, an algorithm for metagenomic SNP (mSNP) calling was developed. This approach is similar to SNP calling for a single sequenced genome and uses heuristically selected parameters. Essentially, the mapping of a single metagenomic readset to a reference genome catalogue yields an alignment file (in BAM format) containing the coverage profile of each of the genomes. In sufficiently covered genomes (with MEC $\geq 0.5$ and mean coverage $\geq 5 \times$ ), only the nucleotide positions with coverage $\geq 4 \times$ are considered. For each such position, a consensus letter is determined using ad hoc scripts as a letter supported by the highest number of reads (which should not be less than 4); if two major alleles are supported by an identical number of reads, one allele is randomly selected. A metagenomic SNP is defined as a position in a consensus sequence with an alternate letter compared to the reference genome. As a genomic dissimilarity measure of $E$. coli species between two metagenomes, we measured the Hamming distance between the two respective consensus sequences adjusted based on the coverage information; only the genome fragments belonging to core genes were used. Specifically during this analysis focused on metagenomic SNPs, each metagenome was mapped to a reference genome of the E. coli strain K-12 alone. To compare the $E$. coli genomes with the metagenomic profiles of $E$. coli, each isolated genome was randomly fragmented into reads (mean targeted coverage $100 \times$ ) before being subjected to identical consensus sequence identification (mapping to a reference genome catalogue, etc.).

\section{Selection of $E$. coli pangenome and accessory genes in the global gene catalogue}

A representative set of 81 diverse $E$. coli genomes was formed (among them, 28 genomes belonged to commensal strains and 43 genomes belonged to pathogenic strains, including 4 known CD-associated strains [23, 42, $43,82]$ and 10 strains previously isolated from Russian $\mathrm{CD}$ patients from the examined cohort [38]) (Additional file 1: Table S9). Using this set, gene orthology groups (OGs) were constructed using OrthoMCL [83] by $50 \%$ identity of amino acid sequences. As some of the produced OGs were divided by the clustering algorithm into multiple groups due to the incompleteness of the gene sequences, to compensate for this effect, the potentially divided groups were merged using the following heuristic: for each two OGs A and B, pairwise BLASTp alignments of a gene from A to a gene from $B$ were considered for such possible pairs; the score $\mathrm{S}=$ (\% identity) $\times(\%$ match) was computed for each pair; if the average $\mathrm{S}$ across all gene pairs exceeded $6400(80 \times 80)$, the OGs were merged into a single OG.

The nucleotide sequences of all genes of the obtained OGs were aligned against the $3.3 \mathrm{mln}$ gut microbial reference gene catalogue to yield highly similar matches (similarity criterion: $>80 \%$ identity and $>80 \%$ of both subject and query sequence lengths). The obtained 9125 matching genes in the catalogue belonged to 5598 OGs; these genes were further used as a template to assess the presence of the $E$. coli pangenome in the gut metagenomes (the detailed statistics used for the pangenome construction are described in Additional file 1: Table S10). Core genes (genes occurring in all 81 genomes) were subtracted from the pangenome to yield a set of $E$. coli accessory genes (AG) (2993 OGs).

Virulence-associated genes were identified among the accessory genes of $E$. coli by aligning the sequences of the latter to a published list of $76 \mathrm{E}$. coli virulence-associated genes [84] (BLASTn search was performed with the following thresholds: e-value $<1 \mathrm{E}-5$, sequence coverage $>$ $80 \%$, identity $>80 \%$ ). The search yielded 17 genes listed in the Additional file 1: Table S11.

\section{Profiling of $E$. coli gene content in metagenomes and genomes}

The gene content of $E$. coli in the gut metagenomes was estimated in the form of a binary vector of the presence/ absence of each gene included in the pangenome of the species. A gene was considered present if at least $1 \mathrm{read}$ was mapped to the gene during the mapping of all reads to the reference gene catalogue. Here, to adjust for variation in the sequencing coverage and $E$. coli relative abundance across the metagenomes, for each metagenome, a random subsampling was simulated such that the total number of reads mapped to the pangenome was 80,000 . Metagenomes with a lower number of pangenomic reads or less than $50 \%$ coverage of the pangenome were not considered in the analysis of the pangenome and accessory genome. The accessory gene (AG) profile was obtained from the pangenome presence profile by filtering genes corresponding to the core genome. A pangenome and accessory profiles were also produced for genomes in the same format based on the alignment of the genomes against the reference gene catalogue [40] using BLASTn (similarity criterion: > 80\% 
identity for $>80 \%$ of the gene length). The pairwise dissimilarity between the AG profiles was calculated using a binary metric (using the function dist in $\mathrm{R}$ package stats). Hierarchical clustering was performed using the average method.

During the stage of refining the orthology groups specific to Clade 1, the candidate signature OGs of Clade 1 with an OG with an identical function description detected in $\geq 20 \%$ of the AG profiles in Clade 2 were excluded. Here, the OG similarity score was defined as a product of the percent sequence identity and percent query matching length averaged over all possible pairs of genes between the two groups.

\section{Alternative profiling of $E$. coli gene content}

To validate the results of the clustering based on the AG profiles, we used PanPhlAn tool [49], which maps the metagenomic reads against the reference genomes of the target species and then, based on the gene coverage levels, reconstructs the unique gene set of a strain present in the sample. The resulting gene content of $E$. coli in the metagenome is also in the form of a binary vector of the presence/absence of each gene family. The pre-processed database of the E. coli pangenome contained 34,881 gene families. The threshold for a gene family to be considered present in the final presence/absence gene profile was set to a coverage depth $>0.5 \times$ times the mean coverage.

\section{Additional files}

Additional file 1: Additional Tables and Figures. (ZIP $816 \mathrm{~kb}$ )

Additional file 2: Results of subspecies-level analysis of $E$. coli and other microbes in the metagenomes using ConStrains. The columns include: 1 - species name, 2 - number of the detected strain (equal to "NA,insufficient" if no multiple strains were detected), 3 - number of masked samples (equal to 1 if read coverage was insufficient for strain detection), 4 - relative abundance of the strain. (XLSX $167 \mathrm{~kb}$ )

\section{Abbreviations}

AG: Accessory genome; AIEC: Adherent-invasive Escherichia coli; CD: Crohn's disease; IBD: Inflammatory bowel diseases; MEC: Maximum-expected coverage; mSNP: Metagenomic single-nucleotide polymorphism; OG: Orthology group; PCDAl: Pediatric Crohn's Disease Activity Index

\section{Funding}

This study was supported by the Russian Science Foundation (project 16-15-00258).

\section{Availability of data and materials}

The metagenomic reads generated during the study were linked under BioProject PRJNA296946 and deposited in the Sequence Read Archive (SRA) under project ID SRP064372.

\section{Authors' contributions}

VMG conceived the study. VMG, DGA, PLS, ILK, IVM, ESK and TVG supervised the work. MVS, AYK, DNA, EAB and YMB collected the samples. DVR, JPB, VGL, IYK, TAS, AKL, MNS and SYM performed the sample preparations and sequencing. AVP performed the study coordination. AVT, AIM, AVK, DSI, ASP, AVE and BAK analysed the data. AVT, VMG, DGA, AIM, AVE and BAK prepared the manuscript. VMG, AIM and DGA revised the manuscript. All authors have read and approved the final manuscript.

\section{Ethics approval and consent to participate}

This study was approved by the ethics committees of the Moscow Clinical Scientific Center and State Scientific Center of Coloproctology, Moscow, Russian Federation and carried out following the rules of the Declaration of Helsinki of 1975. All patients provided written informed consent for the sample collection and personal data processing.

\section{Consent for publication}

Not applicable.

\section{Competing interests}

The authors declare that they have no competing interests.

\section{Publisher's Note}

Springer Nature remains neutral with regard to jurisdictional claims in published maps and institutional affiliations.

\section{Author details}

${ }^{1}$ Federal Research and Clinical Centre of Physical-Chemical Medicine, Malaya Pirogovskaya 1a, Moscow 119435, Russia. ${ }^{2}$ Moscow Institute of Physics and Technology, 9 Institutskiy per., Dolgoprudny, Moscow Region, Russian Federation141700. ${ }^{3}$ TMO University, 49 Kronverkskiy pr, Saint-Petersburg, Russian Federation197101. "Kazan Federal University, 18 Kremlyovskaya St., Kazan, Russian Federation420008. ${ }^{5}$ Moscow Clinical Scientific Center, 86 Shosse Entuziastov St., Moscow, Russian Federation $111123 .{ }^{6} \mathrm{Clinical}$ and Research Institute of Emergency Children's Surgery and Trauma, 22 Bolshaya Polyanka St., Moscow, Russian Federation 119180. ${ }^{7}$ State Scientific Center of Coloproctology, 2 Salam Adil St., Moscow, Russian Federation123423.

${ }^{8}$ Moscow State University of Medicine and Dentistry, Build. 6, 20 Delegatskaya St., Moscow, Russian Federation127473. ${ }^{9}$ Moscow Regional Research and Clinical Institute, 61/2 Shchepkina str, Moscow, Russian Federation129110. ${ }^{10}$ M.M. Shemyakin - Yu.A. Ovchinnikov Institute of Bioorganic Chemistry of the Russian Academy of Sciences, 16/10 Miklukho-Maklaya St., Moscow, Russian Federation117997.

Received: 20 April 2018 Accepted: 23 November 2018 Published online: 27 December 2018

\section{References}

1. Khor B, Gardet A, Xavier RJ. Genetics and pathogenesis of inflammatory bowel disease. Nature. 2011;474:307-17. https://doi.org/10.1038/ nature10209.

2. Cui B, Feng $Q$, Wang $H$, Wang M, Peng Z, Li P, et al. Fecal microbiota transplantation through mid-gut for refractory Crohn's disease: safety, feasibility, and efficacy trial results. J Gastroenterol Hepatol. 2015;30:51-8. https://doi.org/10.1111/jgh.12727.

3. Suskind DL, Brittnacher MJ, Wahbeh G, Shaffer ML, Hayden HS, Qin X, et al. Fecal microbial transplant effect on clinical outcomes and fecal microbiome in active Crohn's disease. Inflamm Bowel Dis. 2015;21:556-63. https://doi. org/10.1097/MIB.0000000000000307.

4. Paramsothy S, Paramsothy R, Trainee G, Rubin DT, Kamm MA, Kaakoush NO, et al. Faecal Microbiota Transplantation for Inflammatory Bowel Disease: A Systematic Review and Meta-analysis. J Crohns Colitis. 11(10):1180-1199.

5. Manichanh C, Rigottier-Gois L, Bonnaud E, Gloux K, Pelletier E, Frangeul L, et al. Reduced diversity of faecal microbiota in Crohn's disease revealed by a metagenomic approach. Gut. 2006;55:205-11. https:/doi.org/10.1136/gut. 2005.073817.

6. Frank DN, St. Amand AL, Feldman RA, Boedeker EC, Harpaz N, Pace NR. Molecular-phylogenetic characterization of microbial community imbalances in human inflammatory bowel diseases. Proc Natl Acad Sci. 2007;104:13780-5. https://doi.org/10.1073/pnas.0706625104.

7. Morgan XC, Tickle TL, Sokol H, Gevers D, Devaney KL, Ward DV, et al. Dysfunction of the intestinal microbiome in inflammatory bowel disease and treatment. Genome Biol. 2012;13:R79. https://doi.org/10.1186/gb-2012 13-9-r79.

8. Pascal V, Pozuelo M, Borruel N, Casellas F, Campos D, Santiago A, et al. A microbial signature for Crohn 's disease; 2017. p. 813-22. 
9. Erickson AR, Cantarel BL, Lamendella R, Darzi Y, Mongodin EF, Pan C, et al. Integrated metagenomics/Metaproteomics reveals human host-microbiota signatures of Crohn's disease. PLoS One. 2012;7:e49138. https://doi.org/10. 1371/journal.pone.0049138.

10. Nielsen HB, Almeida M, Juncker AS, Rasmussen S, Li J, Sunagawa S, et al. Identification and assembly of genomes and genetic elements in complex metagenomic samples without using reference genomes. Nat Biotechnol. 2014:32:822-8 https://doi.org/10.1038/nbt.2939\%5Cnhttp://www.cbs.dtu.dk/ databases/CAG/.

11. Norman JM, Handley SA, Baldridge MT, Droit L, Liu CY, Keller BC, et al. Disease-specific alterations in the enteric virome in inflammatory bowel disease. Cell. 2015;160:447-60. https://doi.org/10.1016/j.cell.2015.01.002.

12. Pérez-Brocal V, García-López R, Nos P, Beltrán B, Moret I, Moya A. Metagenomic analysis of Crohn's disease patients identifies changes in the Virome and microbiome related to disease status and therapy, and detects potential interactions and biomarkers. Inflamm Bowel Dis. 2015;21:2515-32. https://doi.org/10.1097/MIB.0000000000000549.

13. Lewis JD, Chen EZ, Baldassano RN, Otley AR, Griffiths AM, Lee D, et al. Inflammation, antibiotics, and diet as environmental stressors of the gut microbiome in pediatric Crohn's disease. Cell Host Microbe. 2015;18: 489-500.

14. Gevers D, Kugathasan S, Denson L A, Vázquez-Baeza Y, Van Treuren W, Ren $B$, et al. the treatment-naive microbiome in new-onset Crohn's disease. Cell Host Microbe 2014;15:382-392. doi:https://doi.org/10.1016/j. chom.2014.02.005.

15. Zhang C, Cleveland K, Schnoll-Sussman F, McClure B, Bigg M, Thakkar P, et al. Identification of low abundance microbiome in clinical samples using whole genome sequencing. Genome Biol. 2015;16:265. https://doi.org/10. 1186/s13059-015-0821-z.

16. Welch RA, Burland V, Plunkett G, Redford P, Roesch P, Rasko D, et al. Extensive mosaic structure revealed by the complete genome sequence of uropathogenic Escherichia coli. Proc Natl Acad Sci U S A. 2002;99:17020-4. https://doi.org/10.1073/pnas.252529799.

17. Darfeuille-Michaud A, Neut C, Barnich N, Lederman E, Di Martino P, Desreumaux $P$, et al. Presence of adherent Escherichia coli strains in ileal mucosa of patients with Crohn's disease. Gastroenterology. 1998; 115:1405-13.

18. Lapaquette P, Glasser A-L, Huett A, Xavier RJ, Darfeuille-Michaud A. Crohn's disease-associated adherent-invasive E. coli are selectively favoured by impaired autophagy to replicate intracellularly. Cell Microbiol. 2010;12:99_ 113. https://doi.org/10.1111/j.1462-5822.2009.01381.x

19. Rolhion N, Darfeuille-Michaud A. Adherent-invasive Escherichia coli in inflammatory bowel disease. Inflamm Bowel Dis. 2007;13:1277-83. https:// doi.org/10.1002/ibd.20176.

20. Palmela C, Chevarin C, Xu Z, Torres J, Sevrin G, Hirten R, et al. Adherent-invasive Escherichia coli in inflammatory bowel disease. Gut. 2018;67:574-87.

21. Martinez-Medina M, Aldeguer X, Lopez-Siles M, González-Huix F, López-Oliu C, Dahbi G, et al. Molecular diversity of Escherichia coli in the human gut: new ecological evidence supporting the role of adherent-invasive E. coli (AIEC) in Crohn's disease. Inflamm Bowel Dis. 2009;15:872-82

22. Haberman Y, Tickle TL, Dexheimer PJ, Kim M-O, Tang D, Karns R, et al. Pediatric Crohn disease patients exhibit specific ileal transcriptome and microbiome signature. J Clin Invest. 2014;124:3617-33. https://doi.org/10 $1172 / J C 175436$

23. Miquel S, Peyretaillade E, Claret L, de Vallée A, Dossat C, Vacherie B, et al. Complete genome sequence of Crohn's disease-associated adherentinvasive E. coli strain LF82. PLoS One. 2010;5. https://doi.org/10.1371/journal. pone.0012714

24. Tyakht AV, Kostryukova ES, Popenko AS, Belenikin MS, Pavlenko AV, Larin AK, et al. Human gut microbiota community structures in urban and rural populations in Russia. Nat Commun. 2013;4:2469. https://doi.org/10.1038/ncomms3469.

25. La Carbona S, Sauvageot N, Giard J-C, Benachour A, Posteraro B, Auffray Y, et al. Comparative study of the physiological roles of three peroxidases (NADH peroxidase, alkyl hydroperoxide reductase and thiol peroxidase) in oxidative stress response, survival inside macrophages and virulence of enterococcus faecalis. Mol Microbiol. 2007;66:1148-63. https://doi.org/10. 1111/j.1365-2958.2007.05987.x

26. Palmer KL, Godfrey P, Griggs A, Kos VN, Zucker J, Desjardins C, et al. Comparative Genomics of Enterococci: Variation in Enterococcus faecalis, Clade Structure in E. faecium, and Defining Characteristics of E. gallinarum and E. casseliflavus. MBio. 2012;3:e00318-1. https://doi.org/10.1128/mBio. 00318-11.

27. Lobatón T, Hoffman I, Vermeire S, Ferrante M, Verhaegen J, Van Assche G. Aeromonas species: an opportunistic enteropathogen in patients with inflammatory bowel diseases? A single center cohort study. Inflamm Bowel Dis. 2015;21:71-8. https://doi.org/10.1097/MIB.0000000000000247.

28. Ohkusa T, Okayasu I, Ogihara T, Morita K, Ogawa M, Sato N. Induction of experimental ulcerative colitis by fusobacterium varium isolated from colonic mucosa of patients with ulcerative colitis. Gut. 2003;52:79-83. https://doi.org/10.1136/gut.52.1.79.

29. Finegold SM, Song Y, Liu C, Hecht DW, Summanen P, Könönen E, et al. Clostridium clostridioforme: a mixture of three clinically important species. Eur J Clin Microbiol Infect Dis. 2005;24:319-24. https://doi.org/10.1007/ s10096-005-1334-6.

30. Devkota S, Wang Y, Musch MW, Leone V, Fehlner-Peach $H$, Nadimpalli A, et al. Dietary-fat-induced taurocholic acid promotes pathobiont expansion and colitis in II10-/- mice. Nature. 2012;487:104. https://doi.org/10.1038/ nature11225.

31. Biarc J, Nguyen IS, Pini A, Gossé F, Richert S, Thiersé D, et al. Carcinogenic properties of proteins with pro-inflammatory activity from Streptococcus infantarius (formerly S.bovis). Carcinogenesis. 2004;25:1477-84. https://doi. org/10.1093/carcin/bgh091.

32. Hall AB, Yassour M, Sauk J, Garner A, Jiang X, Arthur T, et al. A novel Ruminococcus gnavus clade enriched in inflammatory bowel disease patients. Genome Med. 2017;9:103. https://doi.org/10.1186/s13073-0170490-5.

33. Wang W, Chen L, Zhou R, Wang X, Song L, Huang S, et al. Increased proportions of Bifidobacterium and the lactobacillus group and loss of butyrate-producing Bacteria in inflammatory bowel disease. J Clin Microbiol. 2014;52:398-406. https://doi.org/10.1128/JCM.01500-13.

34. Willing BP, Dicksved J, Halfvarson J, Andersson AF, Lucio M, Zheng Z, et al. A Pyrosequencing Study in Twins Shows That Gastrointestinal Microbial Profiles Vary With Inflammatory Bowel Disease Phenotypes. Gastroenterology. 2010;139:1844-1854.e1.

35. Dubinkina VB, Tyakht AV, Odintsova VY, Yarygin KS, Kovarsky BA, Pavlenko $A V$, et al. Links of gut microbiota composition with alcohol dependence syndrome and alcoholic liver disease. Microbiome. 2017;5:141. https://doi. org/10.1186/s40168-017-0359-2.

36. Fujimoto T, Imaeda H, Takahashi K, Kasumi E, Bamba S, Fujiyama Y, et al. Decreased abundance of Faecalibacterium prausnitzii in the gut microbiota of Crohn's disease. J Gastroenterol Hepatol. 2013;28:613-9. https://doi.org/ 10.1111/jgh.12073.

37. Machiels K, Joossens M, Sabino J, De Preter V, Arijs I, Eeckhaut V, et al. A decrease of the butyrate-producing species Roseburia hominis and Faecalibacterium prausnitzii defines dysbiosis in patients with ulcerative colitis. Gut. 2014;63:1275-83. https://doi.org/10.1136/gutjnl-2013-304833.

38. Rakitina DV, Manolov Al, Kanygina AV, Garushyants SK, Baikova JP, Alexeev $D G$, et al. Genome analysis of $E$ coli isolated from Crohn's disease patients. BMC Genomics. 2017;18:544. https://doi.org/10.1186/s12864-017-3917-x.

39. Luo C, Knight R, Siljander H, Knip M, Xavier RJ, Gevers D. ConStrains identifies microbial strains in metagenomic datasets. Nat Biotechnol. 2015. https://doi.org/10.1038/nbt.3319.

40. Qin J, Li R, Raes J, Arumugam M, Burgdorf KS, Manichanh C, et al. A human gut microbial gene catalogue established by metagenomic sequencing. Nature. 2010;464:59-65. https://doi.org/10.1038/nature08821.

41. Eaves-Pyles T, Allen CA, Taormina J, Swidsinski A, Tutt CB, Eric Jezek G, et al. Escherichia coli isolated from a Crohn's disease patient adheres, invades, and induces inflammatory responses in polarized intestinal epithelial cells. Int J Med Microbiol. 2008;298:397-409.

42. Krause DO, Little AC, Dowd SE, Bernstein CN. Complete genome sequence of adherent invasive escherichia coli UM146 isolated from ileal crohn's disease biopsy tissue. J Bacteriol. 2011;193:583.

43. Clarke DJ, Chaudhuri RR, Martin HM, Campbell BJ, Rhodes JM, Constantinidou C, et al. Complete genome sequence of the crohn's disease-associated adherent-invasive Escherichia coli strain HM605. J Bacteriol. 2011;193:4540.

44. Ranjit DK, Endres JL, Bayles KW. Staphylococcus aureus CidA and LrgA proteins exhibit Holin-like properties. J Bacteriol. 2011;193:2468-76. https:// doi.org/10.1128/JB.01545-10.

45. Kim Y, Wang X, Ma Q, Zhang X-S, Wood TK. Toxin-antitoxin Systems in Escherichia coli influence biofilm formation through YjgK (TabA) and fimbriae. J Bacteriol. 2009;191:1258-67. https://doi.org/10.1128/JB.01465-08. 
46. Wiggins R, Hicks SJ, Soothill PW, Millar MR, Corfield AP. Mucinases and sialidases: their role in the pathogenesis of sexually transmitted infections in the female genital tract. Sex Transm Infect. 2001;77:402-8. https://doi.org/10. 1136/sti.77.6.402

47. Ng SC, Bernstein CN, Vatn MH, Lakatos PL, Loftus EV, Tysk C, et al. Geographical variability and environmental risk factors in inflammatory bowel disease. Gut. 2013;62:630-49. https://doi.org/10.1136/gutjnl-2012303661.

48. Tailford LE, Owen CD, Walshaw J, Crost EH, Hardy-Goddard J, Le Gall G, et al. Discovery of intramolecular trans-sialidases in human gut microbiota suggests novel mechanisms of mucosal adaptation. Nat Commun. 2015;6: 7624. https://doi.org/10.1038/ncomms8624.

49. Scholz M, Ward DV, Pasolli E, Tolio T, Zolfo M, Asnicar F, et al. Strainlevel microbial epidemiology and population genomics from shotgun metagenomics. Nat Methods. 2016;13:435-8. https://doi.org/10.1038/ nmeth.3802

50. Molodecky NA, Soon IS, Rabi DM, Ghali WA, Ferris M, Chernoff G, et al. Increasing Incidence and Prevalence of the Inflammatory Bowel Diseases With Time, Based on Systematic Review. Gastroenterology. 2012;142:46-54.e42

51. Sommer F, Rühlemann MC, Bang C, Höppner M, Rehman A, Kaleta C, et al. Microbiomarkers in inflammatory bowel diseases : caveats come with caviar. Gut. 2017::1-6.

52. Tyakht AV, Alexeev DG, Popenko AS, Govorun VM. Rural and urban microbiota: To be or not to be. Gut Microbes. 2014;5 https://doi.org/10. 4161/gmic.28685.

53. Eng A, Borenstein E. Taxa-function robustness in microbial communities Microbiome. 2018:6:1-19.

54. Blander JM, Longman RS, Iliev ID, Sonnenberg GF, Artis D. Regulation of inflammation by microbiota interactions with the host. Nat Immunol. 2017; 18:851-60.

55. Chiodini RJ, Van Kruiningen HJ, Thayer WR, Merkal RS, Coutu JA. Possible role of mycobacteria in inflammatory bowel disease. Dig Dis Sci. 1984;29: 1073-9. https://doi.org/10.1007/BF01317078.

56. Zeng MY, Inohara N, Nuñez G. Mechanisms of inflammation-driven bacterial dysbiosis in the gut. Mucosal Immunol. 2017;10:18-26.

57. Viladomiu M, Kivolowitz C, Abdulhamid A, Dogan B, Victorio D, Castellanos $J G$, et al. IgA-coated E. coli enriched in Crohn's disease spondyloarthritis promote T н 17-dependent inflammation. Sci Transl Med. 2017;9:eaaf9655. https://doi.org/10.1126/scitranslmed.aaf9655.

58. Shin N-R, Whon TW, Bae J-W. Proteobacteria: microbial signature of dysbiosis in gut microbiota. Trends Biotechnol. 2015;33:496-503.

59. Kamada N, Chen GY, Inohara N, Núñez G. Control of pathogens and pathobionts by the gut microbiota. Nat Immunol. 2013;14:685-90. https:// doi.org/10.1038/ni.2608.

60. Martin HM, Campbell BJ, Hart CA, Mpofu C, Nayar M, Singh R, et al. Enhanced Escherichia coli adherence and invasion in Crohn's disease and colon cancer. Gastroenterology. 2004;127:80-93.

61. Schnabl B, Brenner DA. Interactions between the intestinal microbiome and liver diseases. Gastroenterology. 2014;146:1513-24.

62. Hoffman LR, Pope CE, Hayden HS, Heltshe S, Levy R, McNamara S, et al. Escherichia coli dysbiosis correlates with gastrointestinal dysfunction in children with cystic fibrosis. Clin Infect Dis. 2014;58:396-9. https://doi.org/10. 1093/cid/cit715

63. Lupp C, Robertson ML, Wickham ME, Sekirov I, Champion OL, Gaynor EC, et al. Host-mediated inflammation disrupts the intestinal microbiota and promotes the overgrowth of Enterobacteriaceae. Cell Host Microbe. 2007;2: 119-29.

64. Imhann F, Bonder MJ, Vich Vila A, Fu J, Mujagic Z, Vork L, et al. Proton pump inhibitors affect the gut microbiome. Gut. 2016;65:740-8. https://doi. org/10.1136/gutjnl-2015-310376.

65. Schaubeck M, Clavel T, Calasan J, Lagkouvardos I, Haange SB, Jehmlich N, et al. Dysbiotic gut microbiota causes transmissible Crohn's disease-like ileitis independent of failure in antimicrobial defence. Gut. 2016;65:225-37. https://doi.org/10.1136/gutjnl-2015-309333.

66. Rabizadeh S, Rhee K-J, Wu S, Huso D, Gan CM, Golub JE, et al. Enterotoxigenic bacteroides fragilis: a potential instigator of colitis. Inflamm Bowel Dis. 2007;13:1475-83. https://doi.org/10.1002/ibd.20265.

67. Darfeuille-Michaud A, Boudeau J, Bulois P, Neut C, Glasser A-L, Barnich N, et al. High prevalence of adherent-invasive Escherichia coli associated with ileal mucosa in Crohn's disease. Gastroenterology. 2004;127:412-21.
68. Wassenaar TM, Gunzer F, Santos ADS, Romeiro FG, Sassaki LY, Rodrigues J, et al. The prediction of virulence based on presence of virulence genes in $E$ coli may not always be accurate. Gut Pathog. 2015;7:15. https://doi.org/10. 1186/s13099-015-0062-4.

69. Kaper JB, Nataro JP, Mobley HLT. Pathogenic Escherichia coli. Nat Rev Microbiol. 2004;2:123-40. https://doi.org/10.1038/nrmicro818.

70. Osborn AM, Bruce KD, Strike P, Ritchie DA, Gadd GM, Hughes MN, et al. Distribution, diversity and evolution of the bacterial mercury resistance (mer) operon. FEMS Microbiol Rev. 1997;19:239-62. https://doi.org/10.1111/j. 1574-6976.1997.tb00300.x.

71. Gullberg E, Albrecht LM, Karlsson C, Sandegren L, Andersson DI. Selection of a Multidrug Resistance Plasmid by Sublethal Levels of Antibiotics and Heavy Metals. MBio. 2014;5:e01918-4. https://doi.org/10.1128/mBio.01918-14.

72. Wirth T, Falush D, Lan R, Colles F, Mensa P, Wieler LH, et al. Sex and virulence in Escherichia coli: an evolutionary perspective. Mol Microbiol. 2006;60:1136-51. https://doi.org/10.1111/j.1365-2958.2006.05172.x.

73. O'Brien CL, Bringer M-A, Holt KE, Gordon DM, Dubois AL, Barnich N, et al. Comparative genomics of Crohn's disease-associated adherent-invasive Escherichia coli. Gut. 2016;66(8):1382-9. https://doi.org/10.1136/gutjnl-2015311059.

74. Didelot X, Nell S, Yang I, Woltemate S, van der Merwe S, Suerbaum S. Genomic evolution and transmission of helicobacter pylori in two south African families. Proc Natl Acad Sci U S A. 2013;110:13880-5. https://doi.org/ 10.1073/pnas.1304681110

75. Patra R, Chattopadhyay S, De R, Ghosh P, Ganguly M, Chowdhury A, et al. Multiple infection and microdiversity among helicobacter pylori isolates in a single host in India. PLoS One. 2012;7:e43370. https://doi.org/10.1371/ journal.pone.0043370.

76. Tomida S, Nguyen L, Chiu B-H, Liu J, Sodergren E, Weinstock GM, et al. Pangenome and comparative genome analyses of propionibacterium acnes reveal its genomic diversity in the healthy and diseased human skin microbiome. MBio. 2013;4:e00003-13. https://doi.org/10.1128/mBio.00003-13.

77. Meador JP, Caldwell ME, Cohen PS, Conway T. Escherichia coli pathotypes occupy distinct niches in the mouse intestine. Infect Immun. 2014;82:19318. https://doi.org/10.1128/IAl.01435-13.

78. Dubinkina VB, Ischenko DS, Ulyantsev VI, Tyakht AV, Alexeev DG. Assessment of k-mer spectrum applicability for metagenomic dissimilarity analysis. BMC Bioinformatics. 2016;17:38. https://doi.org/10.1186/s12859-0150875-7.

79. Yarygin K, Tyakht A, Larin A, Kostryukova E, Kolchenko S, Bitner V, et al. Abundance profiling of specific gene groups using precomputed gut metagenomes yields novel biological hypotheses. PLoS One. 2017;12: e0176154. https://doi.org/10.1371/journal.pone.0176154.

80. Qin J, Li Y, Cai Z, Li S, Zhu J, Zhang F, et al. A metagenome-wide association study of gut microbiota in type 2 diabetes. Nature. 2012;490:5560. https://doi.org/10.1038/nature11450.

81. The Human Microbiome Project Consortium. Structure, function and diversity of the healthy human microbiome. Nature. 2012;486:207-14. https://doi.org/10.1038/nature11234.

82. Nash JHE, Villegas A, Kropinski AM, Aguilar-Valenzuela R, Konczy P, Mascarenhas M, et al. Genome sequence of adherent-invasive Escherichia coli and comparative genomic analysis with other $\mathrm{E}$. coli pathotypes. BMC Genomics. 2010;11:1-15.

83. Li L, Stoeckert CJ, Roos DS. OrthoMCL: identification of ortholog groups for eukaryotic genomes. Genome Res. 2003;13:2178-89. https://doi.org/10.1101/ gr.1224503

84. Joensen KG, Scheutz F, Lund O, Hasman H, Kaas RS, Nielsen EM, et al. Realtime whole-genome sequencing for routine typing, surveillance, and outbreak detection of verotoxigenic Escherichia coli. J Clin Microbiol. 2014; 52:1501-10. 